\title{
La Competencia En Comunicación Lingüística De Estudiantes Que Inician La Carrera Universitaria. Un Estudio Empírico
}

\section{Competence In Communication, Linguistics Students Who Start College Career. An Empirical Study}

Marcelo Mantilla Falcón¹, Mery Esperanza Ruiz Guajala², César Medardo Mayorga Abril ${ }^{3}$.

1. Marcelo Mantilla Falcón: Doctor en Ciencias de la Educación, Magíster en Docencia Universitaria y Administración Educativa. Docente en la Facultad de Contabilidad y Auditoría de la Universidad Técnica de Ambato. luismmantilla@uta.edu.ec

2. Mery Esperanza Ruiz Guajala, Economista, Magíster en Costos y Gestión Financiera. Docente en la Facultad de Contabilidad y Auditoría de la Universidad Técnica de Ambato.meryeruiz@uta.edu.ec

3. César Medardo Mayorga Abril, Doctor en Contabilidad y Auditoría, Magíster en Costos y Gestión Financiera. Docente en la Facultad de Contabilidad y Auditoría de la Universidad Técnica de Ambato.cesarmmayorga@uta.edu.ec

\section{Resumen}

Esta investigación analiza la competencia en Comunicación Lingüística de los estudiantes que inician la carrera universitaria. Busca demostrar que, luego de la ejercitación de actividades pedagógicas en lenguaje y comunicación intra-aula, los estudiantes han mejorado significativamente la competencia lingüística, además, busca caracterizar la fenomenología de la competencia tomando variables como: carrera, sexo, rendimiento académico, edad, prácticas pedagógicas, entre otras. El estudio se lo realizó con 103 estudiantes que iniciaron la formación universitaria de las carreras de Contabilidad, Economía y Finanzas de la Universidad Técnica de Ambato, Ecuador. La investigación es de carácter descriptivo, no experimental, longitudinal, con muestreo no probabilístico. Las evidencias de correlaciones de las dimensiones de la competencia demuestran un $\mathrm{r}$ de Pearson de 0,9517 , resultado altamente significativo, concluyéndose que la práctica pedagógica contribuye directamente al fortalecimiento de la competencia en Comunicación Lingüística y les prepara para el desempeño a lo largo de su vida.

Palabras clave: Competencia, comunicación, comprensión oral, comprensión escrita, interacción oral

\begin{abstract}
This research analyzes the linguistic communication competence of students entering college. Seeks to demonstrate that, after the exercise of educational activities in language and communication intra-classroom, students have significantly improved language skills also seeks to characterize the phenomenology of competition taking variables such as race, sex, academic achievement, age, pedagogical practices, among others. The study was made with 103 university students starting training races Accounting, Economics and Finance from the Technical University of Ambato, Ecuador. The research is not
\end{abstract}




\section{Introducción}

El inicio de la carrera universitaria exige el dominio de varias competencias, entre ellas la competencia en comunicación lingüística la misma que será un eje transversal a lo largo de su vida, cabe entonces preguntarse, ¿Cuál es dominio de la competencia con la que los estudiantes inician una carrera universitaria? La respuesta a esta interrogante orientará el trabajo pedagógico y didáctico del staff docente de manera pluri y multidisciplinaria orientada a una formación integrada y holística preparándole al estudiante para su desempeño a lo largo de su vida. Los informes PISA, (OCDE, 2014) no son alentadores para la comunidad educativa latinoamericana en el área de lenguaje, por lo tanto es sustancial diagnosticar el manejo y dominio de esta competencia en la formación universitaria.

Para la universidad actual, cada vez más centrada en la atención del estudiante como persona que se construye en el proceso de aprendizaje profesional, la formación humanística de profesionales competentes $\mathrm{y}$ comprometidos con el desarrollo social constituye una preocupación y un motivo del que ocuparse (González \& González, 2008). Este es el compromiso que tienen los centros de educación superior con la sociedad y la humanidad. La sociedad no evoluciona sin la educación.

La formación en competencias profesionales es uno de los objetivos esenciales de la universidad actual, ese es su compromiso pragmático y hasta moral; sin embargo, es interesante destacar que el interés por su conocimiento, estudio y profundización no surge, precisamente en la universidad, sino en el mundo del trabajo, aproximadamente por la década de los 1970 `s, los primeros estudios que aparecen son los de McClelland (1973), refutando los indicadores del Cociente Intelectual y aseverando que las personas no son solo inteligencia. Otro autor muy conocedor y estudioso del tema es Mertens (1997) y junto a él otros autores comienza una búsqueda orientada al hallazgo de criterios científicos explicativos de la eficiencia de las personas en el desempeño laboral.

Pronto muchos investigadores volcaron su atención al estudio, caracterización, descripción y análisis de la formación por 
competencia (Spencer \& Spencer, 1993) (Woodruff, 1993), (Le Boterf, 2001), (Bunk, 1994), (Corominas, 2001), tanto que los conceptos fueron diseminados, de tal manera que ahora, el término competencia es un constructo polisémico altamente diversificado. Sin embargo, la mayoría tiene elementos en común.

Para efectos de esta introducción se toma el concepto de Le Boterf (2001) para quien "Una persona competente es una persona que sabe actuar de manera pertinente en un contexto particular eligiendo y movilizando un equipamiento doble de recursos: recursos personales (conocimientos, saber hacer, cualidades, cultura, recursos emocionales, etc.) y recursos de redes (bancos de datos, redes documentales, redes de experiencia especializada, etcétera)".

Desde el punto de vista educativo autores como Tobon (2010) enfoca las competencias desde el ámbito del pensamiento complejo y explica que "no son tareas, conductas ni funciones, aspectos referidos exclusivamente a acciones puntuales y observables de las personas. En el enfoque socioformativo, las competencias son actuaciones integrales de las personas ante actividades y problemas del contexto con ética e idoneidad, en tanto articulan los saberes (saber ser, saber conocer y saber hacer) con el manejo de las situaciones externas del contexto, asumiendo los cambios y la certidumbre con autonomía y creatividad" (pág. 33).
Con estos antecedentes, las competencias se han clasificado de tal manera que ya no es posible quedarse con una universal, sino que cada autor lo divide de acuerdo a su lógica, a su realidad y a sus necesidades, pero lo cierto es que la mayoría lo hacen en competencias genéricas y específicas (Proyect Tuning, 2003).

Las competencias genéricas (transversales, comunes a todas las profesiones). En estas competencias se incluyen elementos de orden cognitivo y de orden motivacional, y se expresan a través de las denominadas:

-Competencias instrumentales, de orden metodológico o de procedimiento, tales como la capacidad de análisis y síntesis, de organización y planificación, y de gestión de información.

-Competencias personales, tales como la capacidad para el trabajo en equipo, la habilidad para el manejo de las relaciones interpersonales, el compromiso ético.

-Competencias sistémicas, que se manifiestan en el aprendizaje autónomo, la adaptación a nuevas situaciones, la creatividad y el liderazgo, entre otras.

Específicas (relativas a una profesión determinada) (González \& González, 2008)

\section{Competencia en Comunicación Lingüística}

En esta investigación se aborda el estudio de la competencia en Comunicación Lingüística o sus variantes como "competencia lingüística", "competencia comunicativa", 
"competencia oral", entre otras, cuyos elementos se detallan a continuación:

El concepto de Competencia Lingüística ya fue utilizado por Chomsky en 1965 citado por Padilla, Martínez, Pérez, Rodríguez, \& Miras (2008), definiéndolo como "sistema de reglas que, interiorizadas por el discente, conforman sus conocimientos verbales (expresión) y le permiten entender un número infinito de enunciados lingüísticos (comprensión)", referido a saber utilizar las reglas gramaticales de la lengua. Lyons en 1969, citado por Padilla y otros (2008) definió la Competencia Pragmática como la "capacidad que el discente adquiere de saber usar adecuadamente una lengua, llevándole a conocer los procedimientos no lingüísticos: las necesidades, las intenciones, los propósitos, las finalidades, etc.", referido a saber usar las funciones de la lengua. Hymes, en 1972, definió la Competencia Comunicativa como la "capacidad que el discente adquiere de saber usar con propiedad una lengua llevándole a distinguir las diversas situaciones sociales posibles", referido a saber usar las funciones de la lengua en situaciones comunicativas sociales (Padilla, Martínez, Pérez, Rodríguez, \& Miras, 2008).

Bajo estas consideraciones, la competencia en comunicación se refiere a "la utilización del lenguaje como instrumento de comunicación oral y escrita, de representación, interpretación y comprensión de la realidad, de construcción y comunicación del conocimiento y de organización y autorregulación del pensamiento, las emociones y la conducta" (Ministerio de Educación y Ciencia, 2007).

La competencia en Comunicación Lingüística se refiere a la utilización del lenguaje como instrumento de comunicación oral y escrita, como instrumento de representación, interpretación y comprensión de la realidad, como instrumento de construcción y de comunicación del conocimiento y como instrumento de organización y de autorregulación del pensamiento, de las emociones y de la conducta, y tiene como objetivo final el dominio de la lengua oral y escrita en numerosos contextos (Padilla, Martínez, Pérez, Rodríguez, \& Miras, 2008).

Autores como Canale y Swain (1980), Hymes (1984), Canale (1995); argumentan que la competencia comunicativa está integrada por estas sub competencias, mismas que en palabras de Jiménez (2007) se describen así:

- La competencia lingüística o gramatical, referida al dominio del código lingüístico y el conocimiento práctico de la estructura, elementos y reglas de la lengua en sus diversos niveles: fonético-fonológico, morfosintáctico y léxico-semántico. Con esta competencia se relaciona el conocimiento de las reglas de ortografía, la correcta pronunciación, el vocabulario o las reglas gramaticales.

- La competencia sociocultural y sociolingüística, referida al dominio de la adecuación del lenguaje a la situación comunicativa (participantes en el acto 
comunicativo, conocimientos compartidos, intención comunicativa, reglas de interacción).

- La competencia textual o discursiva, referida a los conocimientos y técnicas necesarias para organizar la información en un texto concreto logrando un discurso coherente y estructurado o reconstruir el sentido de un texto partiendo de sus distintos elementos y realizando las inferencias necesarias.

- La competencia pragmática o estratégica, relacionada con los conocimientos, destrezas y actitudes necesarias para lograr la eficacia en la comunicación.

La competencia en Comunicación Lingüística y sus dimensiones

Aquí se describen los conceptos detallados en el Departamento de Educación del Gobierno Vasco (2007), se entiende por competencia en Comunicación Lingüística la "habilidad para utilizar la lengua, es decir, para expresar e interpretar conceptos, pensamientos, sentimientos, hechos y opiniones a través de discursos orales y escritos y para interactuar lingüísticamente en todos los posibles contextos sociales y culturales".

Esta competencia se subdivide en cinco dimensiones como se detalla a continuación:

La dimensión comprensión oral engloba el "conjunto de habilidades, conocimientos y actitudes básico para la reconstrucción del sentido de aquellos discursos orales necesarios para la realización personal, académica, social y profesional de los alumnos y alumnas al finalizar la Educación Obligatoria". Esta dimensión está ligada, fundamentalmente, a dos ámbitos de uso de la lengua: el de las relaciones interpersonales y el de los medios de comunicación.

\section{La dimensión comprensión escrita} engloba "el conjunto de habilidades, conocimientos y actitudes básico para la reconstrucción del sentido de aquellos textos escritos necesarios para la realización personal, académica, social y profesional de los alumnos y alumnas al finalizar la Educación Obligatoria”. Esta dimensión agrupa habilidades básicas para el tratamiento y selección de la información que son fundamentales en la sociedad del conocimiento.

La dimensión expresión oral engloba "el conjunto de habilidades, conocimientos y actitudes básico para la producción de los discursos orales, fundamentalmente monogestionados, necesarios para la realización personal, académica, social y profesional de los alumnos y alumnas al finalizar la Educación Obligatoria". Esta dimensión exige el dominio de las habilidades específicas que rigen la producción de discursos orales adecuados a diferentes situaciones comunicativas, coherentes en la organización de su contenido y cohesionados.

\section{La dimensión expresión escrita} engloba "el conjunto de habilidades, conocimientos y actitudes básico para la producción de los textos escritos necesarios para 
la realización personal, académica, social y profesional de los alumnos y alumnas al finalizar la Educación Obligatoria”. Estos textos serán variados y diversos, adecuados a una amplia gama de situaciones comunicativas, coherentes en la organización de su contenido y cohesionados textualmente; así como correctos en sus aspectos formales.

La dimensión interacción oral engloba "el conjunto de habilidades, conocimientos y actitudes básico para el diálogo interpersonal, cuyo adecuado desarrollo es necesario para la realización personal, académica, social y profesional de los alumnos y alumnas al finalizar la Educación Obligatoria". Es una dimensión con rasgos específicos, ligados fundamentalmente a la negociación compartida del significado y al respeto a las normas sociocomunicativas.

Finalmente estas dimensiones se dividen en indicadores que se detallan a continuación:

Tabla 1. Dimensiones e indicadores de la Competencia en Comunicación Lingüística COMPETENCIA EN COMUNICACIÓN LINGÜÍSTICA

\begin{tabular}{|c|c|c|c|c|}
\hline $\begin{array}{l}\text { COMPRENSIÓN } \\
\text { ORAL (CO) }\end{array}$ & $\begin{array}{l}\text { COMPRENSIÓN } \\
\text { ESCRITA (CE) }\end{array}$ & $\begin{array}{l}\text { EXPRESIÓN } \\
\text { ORAL (EO) }\end{array}$ & $\begin{array}{l}\text { EXPRESIÓN } \\
\text { ESCRITA (EE) }\end{array}$ & $\begin{array}{l}\text { INTERACCIÓN } \\
\text { ORAL (IO) }\end{array}$ \\
\hline $\begin{array}{l}\text { 1. Identificar el } \\
\text { sentido global de los } \\
\text { textos (IdTO) }\end{array}$ & $\begin{array}{l}\text { 1. Identificar el } \\
\text { sentido global de } \\
\text { textos escritos (IdTE) }\end{array}$ & $\begin{array}{l}\text { 1. Tener en } \\
\text { cuenta las } \\
\text { características } \\
\text { de la situación } \\
\text { de } \\
\text { comunicación } \\
\text { (TEO) }\end{array}$ & $\begin{array}{l}\text { 1. Tener en } \\
\text { cuenta las } \\
\text { características de } \\
\text { la situación de } \\
\text { comunicación } \\
\text { (TEE) }\end{array}$ & $\begin{array}{l}\text { 1. Participar de } \\
\text { manera activa y } \\
\text { adecuada en } \\
\text { intercambios } \\
\text { comunicativos (PIO) }\end{array}$ \\
\hline $\begin{array}{l}\text { 2. Reconocer el } \\
\text { propósito de textos } \\
\text { orales (RTO) }\end{array}$ & $\begin{array}{l}\text { 2. Reconocer } \\
\text { el propósito de } \\
\text { textos escritos } \\
\text { (RTE) }\end{array}$ & $\begin{array}{l}\text { 2. Planificar el } \\
\text { proceso de } \\
\text { producción } \\
\text { (PEO) }\end{array}$ & $\begin{array}{l}\text { 2. Planificar el } \\
\text { proceso de } \\
\text { producción } \\
\text { (PEE) }\end{array}$ & $\begin{array}{l}\text { 2. Respetar las } \\
\text { normas } \\
\text { sociocomunicativas } \\
\text { (RIO) }\end{array}$ \\
\hline $\begin{array}{l}\text { 3. Seleccionar en } \\
\text { textos orales las } \\
\text { informaciones } \\
\text { pertinentes para los } \\
\text { objetivos propuestos } \\
\text { (STO) }\end{array}$ & $\begin{array}{l}\text { 3. Seleccionar } \\
\text { en textos } \\
\text { escritos las } \\
\text { informaciones } \\
\text { pertinentes } \\
\text { para los } \\
\text { objetivos } \\
\text { propuestos } \\
\text { (STE) }\end{array}$ & $\begin{array}{l}\text { 3. Expresarse de } \\
\text { manera } \\
\text { adecuada y } \\
\text { correcta (EEO) }\end{array}$ & $\begin{array}{l}\text { 3. Expresarse de } \\
\text { manera adecuada } \\
\text { y correcta (EEE) }\end{array}$ & $\begin{array}{l}\text { 3. Utilizar estrategias } \\
\text { para mantener la } \\
\text { comunicación y } \\
\text { aumentar su eficacia } \\
\text { teniendo en cuenta } \\
\text { sus causas y/o efectos } \\
\text { (UIO) }\end{array}$ \\
\hline $\begin{array}{l}\text { 4. Interpretar de } \\
\text { manera crítica el } \\
\text { contenido de textos } \\
\text { orales (InTO) }\end{array}$ & $\begin{array}{l}\text { 4. Interpretar } \\
\text { de manera } \\
\text { crítica el } \\
\text { contenido de } \\
\text { textos escritos } \\
\text { (InTE) }\end{array}$ & $\begin{array}{l}\text { 4. Utilizar } \\
\text { estrategias de } \\
\text { control y de } \\
\text { adecuación } \\
\text { (UEO) }\end{array}$ & $\begin{array}{l}\text { 4. Utilizar } \\
\text { estrategias de } \\
\text { control y de } \\
\text { adecuación } \\
\text { (UEE) }\end{array}$ & \\
\hline $\begin{array}{l}\text { 5. Utilizar estrategias } \\
\text { para favorecer la } \\
\text { comprensión de } \\
\text { diversos tipos de } \\
\text { textos orales (UTO) }\end{array}$ & $\begin{array}{l}\text { 5. Utilizar } \\
\text { estrategias para } \\
\text { favorecer la } \\
\text { comprensión } \\
\text { de diversos } \\
\text { tipos de textos } \\
\text { escritos (UTE) }\end{array}$ & & & \\
\hline
\end{tabular}

Fuente: (Gobierno Vasco, 2007) 
Metodología

La presente es una investigación cualicuantitativa de carácter descriptivo, no experimental, longitudinal porque se tomó datos antes y después de concurrido el semestre académico (ex - ante, ex - post).

Los elementos de observación fueron los estudiantes del primer semestre de la Universidad Técnica de Ambato, UTA, Ecuador, en número de 103 estudiantes, con muestreo no probabilístico criterial. En el estudio participaron 39 hombres $(37,9 \%)$ y 64 mujeres $(62,1 \%)$ que pertenecen indistintamente a las tres carreras de formación: Contabilidad, Economía y Finanzas, mismas que en su orden estuvieron representadas por 35,34 y 34 estudiantes respectivamente. Las edades de los participantes fluctuaron entre los 17 y 22 años, siendo los 18 y 19 años los de mayor frecuencia sumando un porcentaje acumulado de $74 \%$ aproximadamente.

Se aplicó el cuestionario que caracteriza a la competencia en Comunicación Lingüística y sus respectivas cinco dimensiones con sus 21 indicadores concentrados en 106 items formulados con la escala de Likert con opciones de frecuencia: siempre (5), casi siempre (4), a veces (3), rara vez (2) y nunca (1). El cuestionario fue adaptado del Ministerio de Educación del País Vasco (Decreto 175/2007) (Gobierno Vasco, 2007).

La prueba de confiabilidad ex - ante tuvo un alfa de Cronbach de 0,941 y ex - post 0,961.

\section{Resultados}

El primer análisis importante es el grado de percepción (autocalificación) de los estudiantes frente a la concepción y dominio de la competencia en Comunicación Lingüística evaluada antes y después de terminado el periodo académico. Puntualmente se le preguntó cuál es el dominio de la competencia en Comunicación Lingüística y los resultados arrojan una diferencia altamente significativa mediante un análisis de Ji cuadrado con un $p<$ 0,000, con 9 grados de libertad y $a$ de 0,05 . La pregunta tuvo escala de Likert con opciones de excelente $=5 \mathrm{y}$ Deficiente $=1$.

Los porcentajes variaron significativamente como se demuestra en la tabla 2.

Tabla 2. Autocalificación de los estudiantes a su competencia lingüista pre-test post-test.

\begin{tabular}{lcccc}
\multicolumn{1}{c}{ CALIFICACIÓN } & \multicolumn{3}{c}{ Inicial } & \multicolumn{2}{c}{ Final } \\
\cline { 2 - 5 } & Frecuencia & Porcentaje & Frecuencia & Porcentaje \\
Regular & 7 & 6,8 & 1 & 1,0 \\
Bueno & 70 & 68,0 & 33 & 32,0 \\
Muy Bueno & 25 & 24,3 & 66 & 64,1 \\
Excelente & 1 & 1,0 & 3 & 2,9 \\
Total & 103 & 100,0 & 103 & 100,0 \\
\hline
\end{tabular}

Fuente: Encuesta a los estudiantes 
En cuanto a la procedencia de los centros educativos bien sea del sector público, privado o fisco-misional (Estado-iglesia), la mayoría, son de instituciones del gobierno, $(80,6 \%)$, del privado $15,5 \%$ y el resto de centros con financiamiento mixto.

$\mathrm{Al}$ analizar la dimensión lectura también arroja evidencias importantes entre las respuestas ex - ante, ex - post, lo que significa que el trabajo intra-aula tuvo sus efectos. El Ji cuadrado es significativo con $p<0,000$ con $1 \mathrm{gl}$. $\mathrm{y} \propto 0,05$. La práctica constante de la lectura y de temas dejados en libertad, de acuerdo a sus gustos e intereses, tuvo efectos positivos.

Del mismo modo se consultó sobre los gustos por la escritura con resultados similares: Ji cuadrado es significativo con $p<0,000$ con 1 gl, $\mathrm{y} \propto 0,05$.

Tabla 3. Criterios de lectura y escritura ex - ante, ex - post

\begin{tabular}{lcccccccc}
\hline PREFERENCIA & \multicolumn{4}{c}{ LEER } & \multicolumn{4}{c}{ ESCRIBIR } \\
\cline { 2 - 10 } & \multicolumn{2}{c}{ ANTES } & \multicolumn{2}{c}{ DESPUÉS } & \multicolumn{2}{c}{ ANTES } & \multicolumn{2}{c}{ DESPUÉS } \\
& $\mathrm{f}$ & $\%$ & $\mathrm{f}$ & $\%$ & $\mathrm{~F}$ & $\%$ & $\mathrm{f}$ & $\%$ \\
Si & 85 & 82,5 & 93 & 90,3 & 55 & 53,4 & 69 & 67,0 \\
No & 18 & 17,5 & 10 & 9,7 & 48 & 46,6 & 34 & 33,0 \\
Total & 103 & 100,0 & 103 & 100,0 & 103 & 100,0 & 103 & 100,0 \\
\hline
\end{tabular}

Fuente: Encuesta a los estudiantes

En lo que a rendimiento académico tiene que ver, es importante verificar los resultados globales con sus calificaciones en una escala de 0 a 10 puntos, comparativamente entre carreras, sus datos se detallan en la tabla 4.

Tabla 4. Estadística descriptiva del rendimiento académico de los estudiantes por carreras en la asignatura de Lenguaje y Comunicación.

\begin{tabular}{lcccc}
\hline \multicolumn{1}{c}{ MEDIDAS } & TOTAL & ECONOMÍA & FINANZAS & CONTABILIDAD \\
\hline Media & 8,27 & 8,06 & 8,13 & 8,62 \\
Error típico & 0,08 & 0,15 & 0,13 & 0,12 \\
Mediana & 8,40 & 8,10 & 8,10 & 8,60 \\
Moda & 8,60 & 8,10 & 7,90 & 8,60 \\
Desviación estándar & 0,83 & 0,89 & 0,79 & 0,71 \\
Varianza de la muestra & 0,68 & 0,78 & 0,62 & 0,50 \\
\hline
\end{tabular}

Fuente: Encuesta a los estudiantes 
La carrera de contabilidad evidencia el mejor puntaje promedio, y tiene la menor dispersión, sin embargo el rango de los datos es el mayor de todas las carreras. Mediante un ANOVA se determina que si hay diferencia estadística significativa entre las tres carreras con un $\alpha$ de 0,05 y un $p<0,008$. $\left(F_{3,0872}=4,9955\right)$.

Numéricamente el rendimiento académico evidenciado en las calificaciones para todo el grupo de estudio es el siguiente:

Tabla 5. Rendimiento académico de los estudiantes al finalizar el semestre.

\begin{tabular}{ccc}
\hline PUNTAJES & F & \% \\
\hline Menor a 7 $*$ & 7 & 6,80 \\
$\mathbf{7}$ a 7,9 & 26 & 25,24 \\
$\mathbf{8}$ a 8,9 & 46 & 44,66 \\
9 a 10 & 24 & 23,30 \\
TOTAL & 103 & 100,00 \\
\hline
\end{tabular}

Fuente: Encuesta a los estudiantes

(*) Significa que son estudiantes que no promovieron directamente el semestre y deben rendir un examen supletorio.

Los valores numéricos de calificaciones es el resultado promedio de los dos parciales que abarca el semestre y debe superar la nota de 7 sobre 10 para ser promovido, aquí, para el recuento, se tomó intervalos numéricos de un punto. Los datos evidencian que el $68 \%$ de los estudiantes tienen muy buen rendimiento académico (entre 8 y 10 puntos) y que son pocos
$(6,8 \%)$ que no alcanzaron el mínimo requerido para su aprobación.

$\mathrm{Al}$ analizar las dimensiones e indicadores de la competencia en Comunicación Lingüística, los datos de tendencia central y dispersión son los siguientes tanto en el pre-test como en el posttest:

Tabla 6. Estadística descriptiva de las dimensiones de la competencia en Comunicación Lingüística.

\begin{tabular}{lccccccccccc}
\hline \multicolumn{1}{c}{ MEDIDAS } & \multicolumn{4}{c}{ PRE-TEST } & \multicolumn{4}{c}{ POST-TEST } \\
\cline { 2 - 11 } Media & CO1 & CE1 & EO1 & EE1 & IO1 & CO2 & CE2 & EO2 & EE2 & IO2 \\
Error típico & 3,31 & 3,28 & 3,38 & 3,54 & 3,72 & 3,74 & 3,67 & 3,84 & 3,89 & 3,99 \\
Mediana & 0,05 & 0,05 & 0,06 & 0,06 & 0,05 & 0,05 & 0,05 & 0,05 & 0,05 & 0,05 \\
Moda & 3,22 & 3,25 & 3,35 & 3,49 & 3,75 & 3,71 & 3,65 & 3,89 & 3,89 & 3,89 \\
Desviación estándar & 3,92 & 3,02 & 2,91 & 3,60 & 3,99 & 3,92 & 3,66 & 3,31 & 3,30 & 3,83 \\
Varianza de la muestra & 0,53 & 0,54 & 0,59 & 0,56 & 0,51 & 0,53 & 0,53 & 0,54 & 0,50 & 0,46 \\
\hline
\end{tabular}

Fuente: Encuesta a los estudiantes 
La correlación entre las cinco dimensiones de la competencia en Comunicación Lingüística deja algunas evidencias importantes, en la tabla 7 se presentan los resultados del pre test y post test:

Tabla 7. Correlaciones entre indicadores del pre test.

\begin{tabular}{|c|c|c|c|c|c|c|c|c|c|c|}
\hline \multirow{6}{*}{ 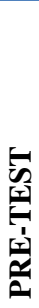 } & \multirow[b]{3}{*}{ CE1 } & \multicolumn{5}{|c|}{ PRE-TEST } & \multicolumn{4}{|c|}{ POST-TEST } \\
\hline & & $\mathrm{CO} 1$ & CE1 & EO1 & EE1 & IO1 & $\mathrm{CO} 2$ & CE2 & $\mathrm{EO} 2$ & EE2 \\
\hline & &, $893^{* *}$ & 1 & & & & & & & \\
\hline & EO1 & ,694** &, $782^{* *}$ & 1 & & & & & & \\
\hline & EE1 &, $746^{* *}$ &, $800^{* *}$ &, $850^{* *}$ & 1 & & & & & \\
\hline & IO1 &, $644^{* *}$ &, $625^{* *}$ &, $691^{* *}$ &, $797^{* *}$ & 1 & & & & \\
\hline & $\mathrm{CO} 2$ &, $409^{* *}$ &, $447^{* *}$ & $455^{* *}$ &, $453^{* *}$ &, $382^{* *}$ & 1 & & & \\
\hline 5 & CE2 &, $339^{* *}$ &, $369^{* *}$ &, $370^{* *}$ &, $386^{* *}$ &, $293^{* *}$ &, $901^{* *}$ & 1 & & \\
\hline 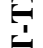 & $\mathrm{EO} 2$ &, $411^{* *}$ &, $414^{* *}$ &, $454^{* *}$ &, $441^{* *}$ &, $376^{* *}$ &, $807^{* *}$ &, $804^{* *}$ & 1 & \\
\hline 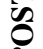 & EE2 &, $416^{* *}$ & $406^{* *}$ &, $454^{* *}$ &, $449^{* *}$ &, $387^{* *}$ &, $763^{* *}$ &, $799^{* *}$ &, $855^{* *}$ & 1 \\
\hline & $\mathrm{IO} 2$ &, $413^{* *}$ &, $396^{* *}$ &, $360^{* *}$ &, $347^{* *}$ &, $352^{* * *}$ &, $650^{* *}$ &, $676^{* *}$ &, $725^{* *}$ &, $765^{\text {** }}$ \\
\hline
\end{tabular}

El cuadro comparativo de los promedios de las dimensiones de la competencia antes y después del semestre es como sigue:

Tabla 8. Promedios de las cinco dimensiones de la competencia en comunicación lingüística.

\begin{tabular}{lcc}
\hline \multicolumn{1}{c}{ DIMENSIONES } & Promedio pre-test & Post-test \\
\hline COMPRENSIÓN ORAL & 3,31 & 3,74 \\
COMPRENSIÓN ESCRITA & 3,28 & 3,67 \\
EXPRESIÓN ORAL & 3,38 & 3,84 \\
EXPRESIÓN ESCRITA & 3,54 & 3,89 \\
INTERACCIÓN ORAL & 3,72 & 3,99 \\
\hline
\end{tabular}

Fuente: Encuesta a los estudiantes

La demostración de la correlación entre los promedios finales de las 5 dimensiones de la competencia en Comunicación Lingüística entre el pre y post test se evidencia en el siguiente gráfico: 


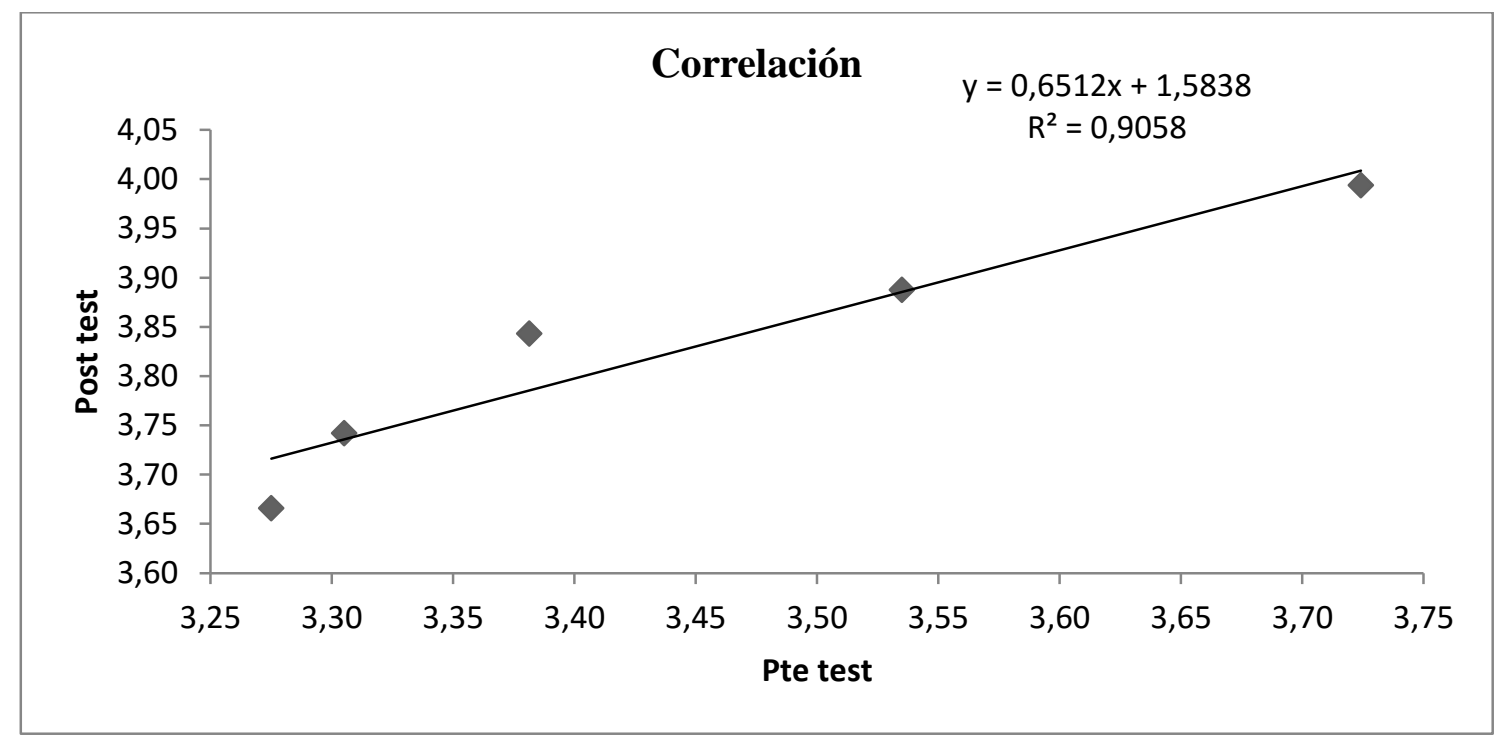

Gráfico 1. Correlación de la competencia en comunicación lingüística ex - ante y ex - post.

\section{Discusión}

La variación de autocalifiación de los estudiantes es significativa al valorar el manejo o dominio de su competencia lingüística antes y después del semestre, esta variación obedece a factores como el método de enseñanza de los docentes, la aplicación de técnicas de lectura, ejercicios de redacción y una amplia reincidencia en talleres de composición literaria, además en la aplicación de discursos auto redactados o memorizados que les permite desenvolverse frente a un público con mayor confianza y seguridad. Al estar empezando la carrera universitaria, los estudiantes toman consciencia que ya no son sujetos de educación media con menores exigencias y alcances. La diferencia es significativa entre las categorías "Bueno" y "Muy Bueno" (ver tabla 2) invirtiéndose profundamente los porcentajes.

La proporcionalidad de mujeres vs. hombres es importante y básicamente las tres carreras son más preferidas por mujeres, en especial, Contabilidad, le sigue en orden de preferencia las Finanzas pero en la carrera de Economía se invierte. Esta es una constante histórica en la facultad.

$\mathrm{El}$ incremento en los indicadores de la competencia se evidencia a través de la práctica pedagógica la misma que permitió potenciar el gusto por la lectura, en unos casos, de manera espontánea y en otros, por obligación o a través de incentivos en notas (calificaciones). El porcentaje en términos positivos varió en un 8\% aproximadamente. De la mano con la lectura va la escritura y este elemento de su formación lingüística tuvo mejores resultados con una diferencia porcentual positiva de aproximadamente el 14\%. El trabajo en aula y autónomo versó sobre redacción y composición en textos de carácter descriptivo (autorretratos, etopeyas, prosopografías, topografías, etc.), expositivos, argumentativos (ensayos) y narrativos (micro-cuentos). 
Los datos comparativos de cada una de las dimensiones de la competencia en Comunicación Lingüística son potencialmente positivos para la aplicación de la prueba posttest. Las variaciones son muy importantes y obedecen al trabajo intra y extra-aula y a la práctica pedagógica diversa a través de la pedagogía activa implementada con el único afán de potenciar el aprendizaje significativo y enseñarles competencias para toda la vida.

El análisis de las correlaciones de Pearson son altamente significativas. La comprensión oral $(\mathrm{CO} 2)$ ex - post presenta la mayor correlación con la Comprensión escrita (CE2) ex - post esto significa que habiéndose transcurrido un semestre de trabajo pedagógico, entender la teoría de la escritura literaria y académica contribuye a la comprensión oral entendida como "la reconstrucción del sentido de aquellos discursos orales necesarios para la realización personal, académica, social y profesional". Concomitantemente la siguiente correlación altamente significativa se da entre las mismas dimensiones pero en fase inicial, es decir antes del semestre (CO1 vs. CE1). Le sigue en orden de importancia la correlación entre la Expresión Oral Expresión Escrita (EO2 vs. EE2) ex - post, evidenciado que mejora la comunicación oral en función de la calidad de la escritura. Por otro lado, la menor correlación evidenciada en el estudio se da entre Interacción Oral (IO1) inicial con la Comprensión Escrita final (CE2) (.293).
Finalmente se evidencia una correlación altamente significativa entre los promedios de las dimensiones de la competencia en Comunicación Lingüística entre el pre test y post test, con un $\mathrm{r}$ de Pearson de 0,9517. Su coeficiente de determinación $\mathrm{r}^{2}$ es de 0,9058 .

\section{Conclusiones}

La correlación de la competencia en comunicación lingüística evaluada al inicio del semestre versus la evaluación al final del semestre es directamente proporcional con un $\mathrm{r}$ de 0,9517 lo que expresa que hay una correlación muy fuerte.

El proceso pedagógico y didáctico aplicado en el aula ha sido determinante para demostrar la variación de los índices de la competencia en comunicación lingüística antes y después del semestre.

Los intereses por la lectura y por la escritura tuvieron diferencias significativas entre los datos iniciales y finales del estudio, los mismos que se asocian a la práctica pedagógica aplicada por el docente.

La percepción inicial de autoevaluación de su competencia lingüística difiere significativamente con la evaluación ex - post de la misma, al concluir el semestre. La segunda evaluación se hace con mayor exactitud y conciencia en función de lo aprendido y de práctica pedagógica asimilada como parte del proceso enseñanza aprendizaje. 
La correlación más significativa se da entre la Comprensión Escrita y Compresión Oral ex - post, esta tiene una relación directa fuerte, su r es de 0,901.

\section{Bibliografía}

1.BUNK, G. P. (1994). La transmisión de las competencias en la formacion $y$ perfeccionamiento profesionales de RFA. Revista Europea de Formacion Profesional(1), 8-14.

2.CANALE, M. (1995). De la competencia comunicativa a la pedagogía comunicativa del lenguaje. En Llobera y otros, Competencia comunicativa. Documentos básicos en la enseñanza de lenguas extranjeras. Madrid: Edelsa.

3.CANALE, M., \& Swain, M. (1980). Fundamentos teóricos de los enfoques comunicativos. La enseñanza y la evaluación de una segunda lengua I y II. Signos. Teoría y práctica de la educación(17-18).

4.COROMINAS, E. (2001). Competencias genéricas en la formación universitaria. Revista de Eduación, 299-321.

5.GOBIERNO VASCO. (2007). Competencia en comunicacion linguistica. Pais Vasco: Departamento de Educación del Gobierno Vasco.

6.GONZÁLEZ, V., \& González, R. (2008). Competencias genéricas y formación profesional: un análisis desde la docencia Universitaria. Revista Iberoamericana de Educación(47), 185-209.

7.HYMES, D. (1984). Vers la competénce de comunication. Paris: Hatier.
8.JIMÉNEZ, J. R. (18 de Septiembre de 2007). La competencia básica de comunicación linguistica. Recuperado el 21 de Septiembre de 2015, de http://www.redes-

cepalcala.org/inspector/DOCUMENTOS $\% 20$ Y\%20LIBROS/COMPETENCIAS/LA\%20C OMPETENCIA\%20LISGUISTICA\%20\%20JOSE\%20RAMON.pdf

9.LE BOTERF, G. (2001). Ingenieria de las competencias. Barcelona: Epise.

MCCLELLAND, D. (1973). Testing for Competencies rather than Intelligence. American Psichologist, 28(1), 1-14.

10.MERTENS, L. (1997). Competencia laboral: sistemas, surgimiento y modelos. Montevideo: Organizacion Internacional del Trabajo (OIT), Centro Interamericano para el Desarrollo del Conocimiento en la Formación Profesional.

11.MINISTERIO DE EDUCACIÓN Y CIENCIA. (2007). REAL DECRETO 1631/2006. Madrid: Ministerio de Educación y Ciencia.

12.OCDE. (2014). Resultados de PISA 2012 en Foco. Lo que los alumnos saben a los 15 años de edady lo que pueden hacer con lo que saben. París: OCDE. 13.PADILLA, D., Martínez, M. C., Pérez, M. T., Rodríguez, C. R., \& Miras, F. (2008). La competencia linguistica como base del aprendizaje. INFAD Revista de Psicologia(1), 177184.

14.PROYECT Tuning. (2003). Tuning Educational Structures in Europe. Informe Final. Proyecto Piloto. Fase 1. Bilbao: Universidad de Deusto. 
15.SPENCER, L. M., \& Spencer, S. M. (1993).

Competence at work: Models for Superior Performance.

Nueva York: Wiley \& Sons.

16.TOBÓN, S. (2010). Formación integral y

Competencias. Pensamiento complejo, currículo, didactica y evaluación. Bogotá D.C: Ecoe Ediciones.

17.WOODRUFF, C. (1993). What is Meant by a

Competency? Development Journal, 14(1), 29-36. 